\title{
Efecto del cambio de patrón de lateralidad a función en grupo e inhibición propioceptiva periodontal en sujetos con guía canina
}

\section{Juan Zegarra Zegarra}

Residente de Rehabilitación Oral. Facultad de Estomatología. Universidad Peruana Cayetano Heredia

Zegarra J. Efecto del cambio de patrón de lateralidad a función en grupo e inhibición propioceptiva periodontal en sujetos con guia canina. Rev Estomatol Herediana 2003;13(1-2) : 19 - 22

RESUMEN

En este estudio se evaluó la influencia muscular en la modificación del patrón de lateralidad de guia canina a función en grupo y la inhibición propioceptiva periodontal en posiciones funcionales y parafuncionales entre personas de 16 a 21 años de edad que presentan normoclusión.

Palabras clave: Guía canina - Función en grupo - Inhibición propioceptiva periodontal Posición funcional - Posición parafuncional.

Effect of the changes of lateral pattern to group function and periodontal propioceptive inhibition in individuals with canine guidance.

ABSTRACT

This study was aimed to evaluate the muscular influence in the modification of lateral patterns of Canine Guidance as compared to Group Function and periodontal propioceptive inhibition in functional and parafunctional positions in 16 to 21 years old individuals with normocelusion.

Key words: Canine guidance - Group function - Inhibition of the periodontal propioception Functional positions - Parafunctional positions.

\section{Introducción}

Existe mucha controversia sobre lo ideal de un esquema oclusal. La guía anterior puede ser categorizada como función de grupo o guía canina, que es esencial para la estética, fonética, la masticación y parafunciones (1). No existe evidencia científica que señale preferencia de un esquema oclusal sobre otro, pero existen autoridades en el campo de la oclusión que están a favor de una guía canina en comparación a una función en grupo (1).

El criterio para evaluar la conveniencia de una posición oclusal es determinar la conveniencia de dicha posición con respecto a su acción sobre el aparato neuromuscular.

Para que haya un estímulo consiente, es necesario que la excitación del sistema nervioso periférico tlegue hasta êt cerebro, si este estímulo nace a partir de la oclusión dentaria, se recepciona por los propioceptores periodontales y es conducido por la raíz sensorial del trigémino ẩ núčleo mesencefálico de este nervio. Desde allí atraviesa estruc- turas reticulares, hasta la corteza sensorial del cerebro, donde es integrado y el individuo hace consiente el estímulo. Una vez producido esto, hay una respuesta de la corteza motora, el impulso motor desciende hasta el núcleo motor del trigémino, de donde sigue la vía descendente motora de ese nervio, el cual producirá la estimulación del grupo muscular (2).

Hay aparatos que pueden rastrear electrónicamente los movimientos mandibulares, un ejemplo de esto es el Cinestesiógrafo MKG (Mandibular Kinesiograph), el cual puede medir cambios mínimos en los movimientos mandibulares. En siglo XVIII Galvani lanzó la hipótesis que la contracción muscular se debía a corriente eléctrica y en 1980 se diseñó el primer electromiógrafo orientado a la investigación dental (3).

La electromiografía se basa en los mecanismos fundamentales de la contracción muscular esquelética, es decir, los procesos de despolarización y repolarización local a nivel de una sinapsis neuromuscular y su propagación por el resto de la fibra muscular (potencial de acción) (4). Los receptores mecánicos localizados a nivel periférico de las raíces dentales son los encargados de cuantificar los contactos realizados por la pieza dentaria y enviar la información al cerebro, el cual ordena cierto tipo de contracción muscular dependiendo del grado de fuerza que necesita el músculo para realizar su función (5). Los contactos de piezas anteriores pueden funcionar como protección en los movimientos excursivos sobre las piezas posteriores y las piezas posteriores pueden proteger a las piezas anteriores de las sobrecargas. Esta relación se podría llamar de mutua protección (6). Miller realizó muchos estudios sobre los tipos de desoclusión, pero no se ha logrado encontrar un patrón de desoclusión repetitivo en la mayoría de personas (7). Lo que si se ha podido determinar es el conocimiento clínico de cómo las fuerzas asociadas con contactos en lado de no trabajo pueden ser destructivas. 
Hay muchos estudios como los de D'amico (12), Beaudreu (6) en los cuales se determina que la guía canina es el contacto ideal en el lado de trabajo en una lateralidad ya que actúa como rompefuerzas y disminuye la actividad muscular de los músculos elevadores. Lucia en 1961 afirmó que los caninos en a oclusión actúan como guardas oropioceptores debido al gran tamaño le su raíz. Korioth (7) añadió que en ratamientos de rehabilitación extensos, e debe elegir la guía canina porque disribuye mejor el estrés, pero sólo si el canino tiene buen soporte. Sin embarso hay otros estudios en los cuales se lemuestra que una función en grupo listribuye mejor las fuerzas ejercidas. 3orromeo (8) también mencionó que la cción muscular ejercida por una funión canina no es significativamente nenor que una función de grupo. Otros nvestigadores como Weinberg (16) y lexander (17) cuestionan el concepto ue la cúspide protectora sea benefiiosa para una relación oclusal. Veinberg (16) encontró que solo dieciueve de cada cien sujetos presentaan guía canina, Alexander opinó que guía canina predisponía a la pérdida e hueso alveolar alrededor de dicha ieza dentaria. También se han hecho studios sobre movilidad dentaria en ersonas con función de grupo con resecto a función canina y se llega a la onclusión que una función de grupo más deseable ya que causa menor ovilidad dentaria (9).

Algunos estudios realizados en el erú $(10,11)$ han mostrado mostraron de sujetos que presentaban patrón de día canina natural tenían mayor activiad muscular que sujetos con función grupo en máximo apretamiento en lusión habitual. Por lo contrario, en áximo apretamiento en lateralidades se contraba mayor actividad muscular en nción de grupo con respecto a guía la nina (10). En un estudio peruano, se contró que la guía canina sin modifición disminuía la actividad muscular omedio al compararla con función de upo natural, además se determinó que a guía canina con modificación prentaba menor actividad muscular que una guía canina sin modificaciones y que una guía canina con modificación tenia mayor actividad muscular que una función de grupo modificada (11).

Por lo expresado anteriormente, es siempre de controversia el tipo de patrón de desoclusión lateral recomendado para la rehabilitación oral y para el tratamiento de los problemas de disfunción del sistema estomatognático y además, es de mayor incógnita como se producirían los cambios en el sistema y que componentes estarían determinando dichos cambios.

El objetivo del presente estudio fue evaluar la influencia de los músculos en la modificación del patrón de lateralidad de guía canina a función en grupo y la inhibición de la sensibilidad propioceptiva peridontal de los caninos y piezas posteriores en posición de reposo, apretamiento en oclusión habitual, apretamiento en lateralidad derecha y masticación unilateral.

\section{Materiales y métodos}

El diseño dei presente estudio fue experimental in vivo, del tipo analítico dado que se realizaron inferencias que asocian el efecto del patrón de lateralidad y la propiocepción en los músculos masetero (M), digástrico (D) y temporal (T). Prospectivo porque los resultados se dieron posterior a la introducción del factor experimental.

El grupo de estudio estuvo com- puesto por cinco sujetos de 16 a 21 años de edad, estudiantes de la Universidad Peruana Cayetano Heredia que presentaban normoclusión y ausencia de alteraciones temporomandibulares. En este grupo el patrón natural de lateralidad fue modificado a función en grupo mediante la colocación de resina compuesta a nivel de premolares y molares sin alterar los contactos oclusales en posiciones funcionales. La inhibición propioceptiva periodontal fue realizada en canino y piezas posteriores mediante técnica de anestesia crestal (técnica a nivel de cresta ósea, orientada hacia los canales nutrícios de Zuckerland, no involucra el ligamento peridontal). Estas modificaciones fueron medidas electromiográficamente a nivel de los músculos: Fascículo anterior del tempo$\mathrm{ral}(\mathrm{T})$, vientre anterior del digástrico (D) y masetero (M) (Fig. 1) en posiciones de apretamiento máximo en lateralidad derecha (AmaxLD), apretamiento máximo en oclusión habitual (AMaxOH), posición postural (Rep), masticación unilateral derecha con alimento blando (MastUD-AB) y duro (MastUD-AD). Por consiguiente se intrơdujeron cuatro factores experimentales: guía canina natural (GCN), guía canina natural más anestesia (GCNa), función en grupo artificial (FGAr) y función en grupo artificial más anestesia (FGAra). El estudio estadístico se realizó mediante un análisis de varianza (ANOVA).

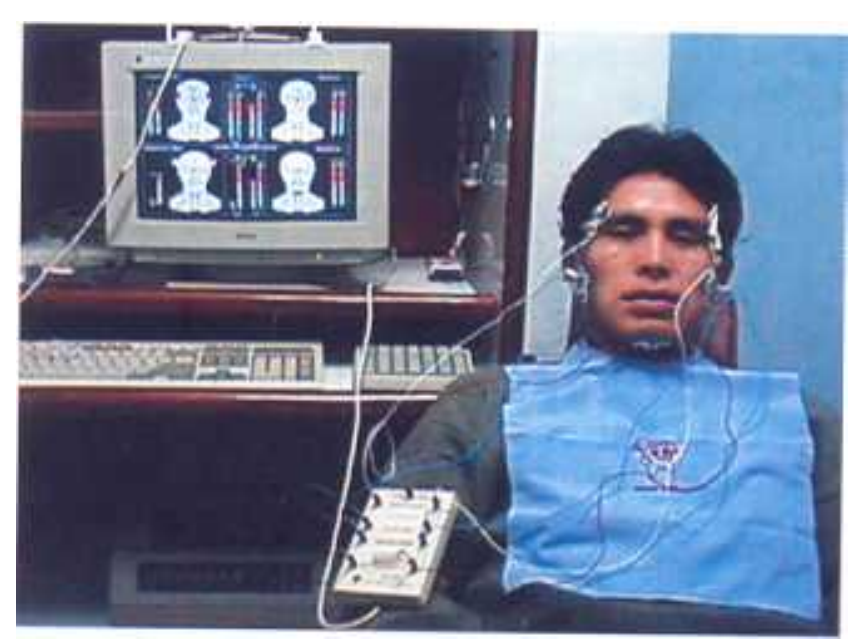

Fig. 1. Toma de medidas electromiográficas. 


\section{Resultados}

Guía canina natural (GCN) a función en grupo artificial (FGAr) en general hubo una tendencia a incrementar la actividad muscular a nivel de las cinco posiciones evaluadas. Es necesario remarcar que en AmaxLD el incremento de la actividad muscular es a nivel de todos los músculos evaluados (Tabla 1).

Guía canina (GCN) a guía canina más anestesia (GCNa) en MastUD promedio hubo una tendencia a disminuir la actividad muscular, esta disminución fue estadísticamente significativa en el masetero derecho y ambos temporales. En posición de reposo, AmaxOH y AmaxLD la actividad fue mayor en GCNa(Tabla 1).

Función en grupo artificial (FGAr) a función en grupo artificial mas anestesia (FGAra) en general hubo una dismi- nución en la actividad muscular, esta disminución fue estadísticamente significativa a nivel de ambos temporales en $\mathrm{AMaxOH}$ y en el temporal derecho así como en ambos digástricos en MastUD-AB (Tabla 2).

Guía canina (GCN) a función en grupo artificial más anestesia (FGAra) la actividad muscular decreció significativamente en el Temporal izquierdo en todas las posiciones evaluadas (Tabla 1).

Guía canina más anestesia (GCNa) a función en grupo artificial más anestesia (FGAra) hubo un incremento en la actividad muscular de todos los músculos en posición de AmaxLD y en MastUD. En reposo y AmaxOH hubo una tendencia a disminuir la actividad muscular (Tabla 2).

Función en grupo artificial (FGAr) a guía canina mas anestesia (GCNa) se en- contró una tendencia a disminuir la actividad muscular a nivel de todas las posiciones evaluadas y en AmaxLD esta disminución se dió a nivel de todos los músculos evaluados (Tabla 2).

\section{Discusión}

En el presente estudio se encontraron mayores niveles de actividad muscular en función en grupo artificial que en guía canina natural, esto en AMaxLD a nivel de todos los músculos evaluados, que confirma que el canino actuaría como un rompefuerzas en movimientos parafuncionales (12).

La transferencia de presión al periodonto es directamente proporcional al área de superficie periodontal. El área periodontal del canino es menor que la suma de áreas periodontales de las piezas posteriores, con lo tanto la transferencia de presión es menor y la

Tabla 1. Modificación del patrón de lateralidad e inhibición propioceptiva

\begin{tabular}{|l|l|l|l|l|l|l|l|l|l|l|l|l|l|l|l|l|l|l|l|}
\hline Grupo & \multicolumn{10}{|c|}{ GCN a FGAr } \\
\hline Pos/mus & MD & MI & TD & TI & DD & DI & MD & MI & TD & TI & DD & DI & MD & MI & TD & TI & DD & DI \\
\hline Rep & + & + & - & + & + & $=$ & - & + & + & - & + & + & $-*$ & + & - & $-*$ & + & + \\
\hline AMaxOH & - & + & - & - & & & + & + & - & - & & & - & - & - & $-*$ & & \\
\hline AMaxLD & + & + & + & + & & & + & + & - & + & & & + & + & - & + & & \\
\hline MastUD & - & - & - & - & + & + & $-*$ & - & $-*$ & - & - & + & - & - & $-*$ & $-*$ & + & + \\
\hline Mast-AB & + & + & + & - & + & + & - & - & - & - & - & + & - & - & - & - & + & + \\
\hline Mast-AD & - & $-*$ & - & $-*$ & + & + & $-*$ & - & $-*$ & $-*$ & - & $+*$ & - & - & - & $-*$ & + & + \\
\hline
\end{tabular}

Tabla 2. Modificación del patrón de lateralidad e inhibición propioceptiva

\begin{tabular}{|l|l|l|l|l|l|l|l|l|l|l|l|l|l|l|l|l|l|l|}
\hline Grupo & \multicolumn{1}{|c|}{ FGAr a FGAra } & \multicolumn{1}{|c|}{ GCNa a FGAra } & \multicolumn{1}{c|}{ FGAra GCNa } \\
\hline Pos/mus & MD & MI & TD & TI & DD & DI & MD & MI & TD & TI & DD & DI & MD & MI & TD & TI & DD & DI \\
\hline Rep & - & + & - & - & - & - & - & $-*$ & - & + & - & - & - & + & - & - & - & - \\
\hline AMaxOH & - & - & $-*$ & $-*$ & & & - & - & - & - & & & + & + & - & - & & \\
\hline AMaxLD & - & + & - & - & & & + & + & + & + & & & - & - & - & - & & \\
\hline MastUD & + & + & - & - & - & - & + & + & + & + & + & - & - & + & - & - & - & - \\
\hline Mast-AB & - & - & $-*$ & - & $-*$ & $-*$ & + & - & + & + & + & - & - & - & - & - & - & + \\
\hline Mast-AD & + & + & + & + & - & - & + & + & + & + & + & + & + & + & - & - & - & - \\
\hline
\end{tabular}

(-) Denota una disminución en la AEMG, (+) denota un aumento en la AEMG

* Cambio estadísticamente significativo

(X)D Músculo derecho

(X)I Músculo izquierdo 
actividad muscular disminuye en comparación a un patrón de lateralidad de función en grupo (13). Esto se debería también a la mayor inervación del canino (14) cuya inervación tiene propioceptores del núcleo trigeminal, así como del núcleo mesencefálico, siendo esta distribución muy diferente a la del resto de piezas dentarias (5). Esto ha sido corroborado en estudios en caninos de animales, donde se encontró que los caninos poseían mayor sensibilidad que otras piezas (15).

La inhibición propioceptiva periodontal mediante aplicación de anestesia a pacientes con guía canina, mostró una reducción en la actividad muscular promedio en posición de MastUD. Esta reducción fue significativa en ambos músculos temporales y también en el masetero derecho. También mostró una disminución en ambos lados en masticación, exceptuando el digástrico izquierdo, donde la actividad muscular auménta. En el caso de reposo, AMaxOH y AmaxLD hubo un aumento en la actividad muscular, especialmente a nivel de los maseteros, mediante anestesia. Esto puede deberse a que se inhibe una respuesta de los propioceptores periodontales evitando así la capacidad de los mismos a realizar un efecto de retroalimentación negativo, lo cual lleva a un aumento de la actividad muscular en movimientos parafuncionales.

En el caso de inhibir la propiocepción periodontal en función en grupo artificial, se mostró una reducción en la actividad muscular en todas las poșiciones evaluadas, siendo esta reducción más significativa al nivel de los temporales en AMaxOH. Lo mismo ocurrió en la evaluación de MastUD-AB y esta reducción significativa fue a nivel de ambos digástricos y temporal derecho. Esto demostraría que los propioceptores de las piezas posteriores tendrían un efecto estimulador sobre la actividad muscular al ser excitados (5).

Cuando se comparó guía canina más anestesia con función en grupo más anestesia, se observó que la actividad muscular aumentó en AmaxLD y MasUD, disminuyendo en AmaxOH y reposo, donde el incremento o reducción de la actividad muscular en cada caso fue dado por los músculos evaluados. Esto indica que la actividad muscular es regida por el patrón de lateralidad así como por la respuesta propioceptiva periodontal (3).

Los resultados de este estudio conducen a sostener que se presenta una mayor actividad muscular al modificar el patrón de lateralidad a función en grupo, esencialmente en posiciones parafuncionales como AmaxLD. Por otro lado, en movimientos parafuncionales como lateralidades, los propioceptores periodontales a nivel de canino presentan la capacidad de disminuir la respuesta muscular.

Del mismo modo la respuesta propio-ceptiva periodontal de las piezas posteriores presentan una capacidad excitatoria sobre la respuesta muscular en las diferentes posiciones evaluadas, especialmente a nivel de los temporales.

En general, la respuesta propioceptiva sobre la actividad muscular se observó en mayor nivel en movimientos parafuncionales.

\section{Referencias}

1. Thorton L. Guía anterior: Función de grupo / función canina. Revisión de la literatura: 74-78.

2. Behsnilian Y. Oclusión y Rehabilitación. 2 edición. Montevideo: Panamericana, 1974: 53-75.

3. Ludin H. Fundamentos Fisipatológicos de la electromiografía. 2 edición. Barcelona: Tosky SA, 1975: 75-87.

4. Linman J, Rithchie A. Clinical Electromyography. Philadelphia: J Ippincott Co. 1970.

5. Byers M, Dong W. Comparison of trigeminal receptor location and structure in the periodontal ligament of different types of teeth from the rat, cat, and monkey. J Comp Neurol 1989;279(1):117-127.

6. Beaudreu D, Guyer S, Lefkowits W. The importance of canine and anterior tooth positions in occlusion. $\mathbf{J}$. Prosteth Dent, 1977; 38(5) 398-410.

7. Korioth TW. Analysis of workingside occlusal contacts. Int. J Prosthodont, 1990; 3(4): 349-355.

8. Borromeo GL. Suvinen TI. Reade PC.
A comparison of the effects of group function and canine guidance interocclusal device on masseter muscle electromyographic activity in normal subjects. J Prosthet Dent 1995; 74(2): 174-80.

9. O'Leary TJ, Shanley DB, Drake RB. Tooth mobility in cuspid-protected and group-function occlusions. J.Prosteth Dent 1972; 27(1): 21-25.

10. Salcedo P, Galarza L. Evaluación de la actividad electromiográfica de los músculos Masetero, fascículo anterior del Temporal en la posición de máximo apretamiento en oclusión habitual y en lateralidad derecha, cuando se altera el patrón de lateralidad de guía canina a función de grupo y viceversa en sujetos con normoclusión dentaria. Lima: UPCH,1998.139 Págs.

11. Otero J. Evaluación de la actividad de los músculos masetero y fascículo anterior del temporal al cambiar los patrones de desoclusión lateral de guía canina a función de grupo y de función de grupo a guía canina, en sujetos con normoclusión dentaria, durante máximo apretamiento en oclusión habitual (Tesis). Lima-Perú: Universidad Peruana Cayetano Heredia; 1998.

12. D'Amico A. Functional occlusion of the natural teeth of man. J prosthet Dent, 1961; 11: 899-915.

13. Manns A. Influence of group function and canine guidance on electromyographic activity of elevator muscle. Universidad de Chile, Facultad de Medicina, Santiago de Chile 1987; 57.

14. Kruger L, Michael F. A single neuron analysis of buccal cavity representation in the sensory trigeminal complex of the cat. Arch Oral Biol, 1962; 7: 491-503.

15. Corbin K, Harrison F. Function of Mesencephalic Root of Fufth Cranial Nerve, J. Neurophysiol, 1940; 3 : 423-435.

16. Weinberg L. A cinematic study of centric and eccentric occlusions. J Prosthet Dent 1964;14:290-293.

17. Alexander $P$. The periodontum and the canine function. J Prosthet Dent 1967:18:571-578. 